\title{
Re-anatomización de un incisivo lateral geminado con talón cuspídeo: Reporte de caso
}

\author{
Re-anatomization of a geminated lateral incisor with talon cusp: Case report \\ Lucia Aguirre-Vela ${ }^{1, a}$, Louise Webb Linares ${ }^{1, b}$
}

\section{RESUMEN}

Los requerimientos estéticos actuales hacen que los profesionales deban estar preparados para ofrecer alternativas de tratamiento rápidas y accesibles al paciente. Este reporte describe el caso de una paciente que presenta un incisivo lateral geminado con talón cuspídeo, el cual es un hallazgo muy raro, y el contralateral sólo con talón cuspídeo.

PALABRAS CLAVE: Anomalías dentarias, resinas compuestas, coronas con frente estético (Fuente: DeCS)

\section{SUMMARY}

The current aesthetic requirements make that the professionals must be prepared to offer alternatives of fast and accessible treatment to the patient. This report describes the case of a patient who presents the left maxillary lateral incisor geminated with talon cusp, which is a very rare found, and the right maxillary lateral incisor just with talon cusp.

KEY WORDS: Tooth abnormalities, composite resins, dental veneers (Source: MeSH).

\section{INTRODUCCIÓN}

Se denomina anomalía dentaria a un desorden de crecimiento o desarrollo en las estructuras anatómicas bucales que resulta en algo diferente de lo normal $(1,2)$. La geminación y el talón lingual son anomalías que pueden generar problemas tanto clínicos como estéticos, por este motivo han recibido distintos tratamientos poco conservadores como exodoncia de la pieza dental para el caso de geminación o el desgaste y posterior endodoncia para el caso del talón lin$\operatorname{gual}(3,4,5,6,7)$.

\footnotetext{
Facultad de Estomatología, Universidad Peruana Cayetano Heredia. Lima, Perú.

Diplomada en Odontología Restauradora y Estética

Magister
} 
El objetivo de este artículo fue relatar un caso poco común con presencia simultanea de geminación y talón lingual en un incisivo lateral superior en dentición permanente, junto al tratamiento al cual estuvo sujeto. Este tratamiento se enfocó en lograr excelentes resultados estéticos y funcionales de manera conservadora y en pocas citas. Así, mediante una carilla directa con el uso de resina compuesta, se le brindó estética a la sonrisa del paciente alcanzando su satisfacción plena.

\section{Geminación}

La geminación dental es una anomalía poco común que puede ocurrir durante el desarrollo del germen dental en la que éste intenta dividirse dando origen a un diente ancho con corona bífida y un conducto radicular $(1,2,3,4,8,9,10)$. Esto puede resultar en la formación de dientes de tamaño y dimensiones iguales o un par. de dientes, de los cuales uno presenta tamaño normal y el otro forma rudimentaria (11)

Esta anomalía presenta una prevalencia fluctuante entre el 0,1 y $1 \%$ presentándose mayormente en dentición primaria. Los dientes afectados con mayor frecuencia son los incisivos y caninos superiores. $(5,6,12)$. Además, parece no haber diferencias en cuanto al género(4,5,12).

La geminación puede desarrollarse durante la morfo-diferenciación del brote dental como resultado de una aberración en el desarrollo del ectodermo y mesodermo $(6,13)$. La etiología de los dientes geminados permanece desconocida; sin embargo, se piensa que en el proceso de la geminación los factores genéticos y ambientales juegan un rol importante $(5,7,11,12,13)$. Según Hattab y Tomazinho, esta anomalía presenta una tendencia hereditaria similar a la macrodoncia o dientes supernumerarios respectivamente; mientras queSpouge, referido por Hattab, sugiere que puede resultar de un trauma durante el brote dental $(7,12)$. Usualmente es encontrado como un rasgo aislado, no asociado a síndromes(12).

Respecto a las características clínicas, la geminación da como resultado 2 coronas parcial o totalmente separadas. El diente anómalo posee una corona grande bífida mostrando un surco de poca profundidad desde la zona incisiva hasta la zona gingival. Radiográficamente, presenta una cámara pulpar agrandada, un conducto y una raíz $(1,9,12,13)$.Sin embargo, Nevi- lle et al., refirieron que los dientes geminados pueden mostrar una corona bífida con dos raíces completamente separadas $(1,14)$.

La geminación es a menudo confundida con la fusión por presentar similares características clínicas (5). Así, Brook y Winter resolvieron la dificultad de decidir si un diente se encontraba fusionado o geminado proponiendo que ambas anomalías sean referidas con un término neutral, como "diente doble" $(15,16)$. Sin embargo, pueden ser usados criterios clínicos y radiográficos para distinguirlas, pues mientras que la fusión es un intento incompleto de dos brotes dentales de unirse formando uno, la geminación es un intento incompleto de un brote dental de dividirse en dos $(6,16)$.Clínicamente, cuando los dientes unidos son contados como uno y hay un conteo total correcto de dientes, se considera geminación; en cambio, si el conteo total de dientes es incorrecto, esto indicaría que es una fusión. Una consideración radiográfica es la diferencia en la configuración radicular vista a menudo en ambas anomalías, en el caso de geminación el diente usualmente presenta sólo un conducto en común, mientras que en el caso de fusión usualmente presenta 2 conductos separados $(2,6,9,16)$.

\section{Talón cuspídeo}

Descrito por primera vez como un proceso con forma de cuerno en la superficie palatina de un incisivo central superior izquierdo (17), no fue hasta 1970 que los talones cuspídeos fueron nombrados asíen la literatura $(10,16,17,18)$. El término "talón cuspídeo" es usado para describir cúspides anómalas de incisivos permanentes que se proyectan prominentemente desde la superficie lingual del diente, puede estar ubicado desde el área del cíngulo o desde la unión esmalte-cemento de un diente anterior, son morfológicamente bien delineadas y se extienden por lo menos hasta la mitad de la distancia entre la unión cemento-esmalte y el borde incisal.Puede afectar dientes deciduos o permanentes $(4,5,8,10,13,16,19)$.

Se han empleado diferentes nombres para describir el mismo rasgo morfológico, entre las que se encuentran cíngulo hiperplásico, cíngulo cuspídeo, cúspide supernumeraria, tubérculo dental, tubérculo lingual, diente en forma de $\mathrm{T}$, cúspide en garra (eagle'stalon), talón cuspídeo palatino o lingual, talón cuspídeo facial (si se presenta en la superficie vestibular), 
y otros (20). Entre éstos, el término "talón cuspídeo" propuesto por Mellor y Ripa en 1970 será empleado para el abordaje de este rasgo morfológico, dejando de lado el término densevaginatusdebido a que se presta a confusión porque esta condición describe principalmente una proyección de esmalte que ocurre en la superficie oclusal de los premolares y que siempre involucra una proyección de la cámara pulpar (20)Mientras que el talón cuspídeo es una estructura que se encuentra compuesta por esmalte, dentina y se puede presentar con la inclusión o no de la cámara pulpar $(5,7,12,13,16,20)$.

La prevalencia del talón cuspídeo varía considerablemente en la población, fluctuando desde el 0,06 hasta el $7,7 \%(5,10,12,16)$. Se presenta con mayor frecuencia en personas de sexo masculino y en la dentición permanente, principalmente en el maxilar superior (92\%), losdientes más frecuentemente afectados son los incisivos laterales (55\%) seguidos por los incisivos centrales (36\%) y los caninos $(5,7,10,12,16)$.

La etiología de esta anomalía es aún desconocida, aunqueestá asociada aun origen multifactorial,con factores genéticos y ambientales(4,5,7,10,12, 16, 19, 20).De manera similar a otras anormalidades en forma, eltalón cuspídeo se origina durante el estadio de morfo-diferenciación dental en elque las células del epitelio interno del órgano del esmalte se pueden plegar hacia afuera y desarrollar hiperplasias focalesa partir de las células periféricas mesenquimales de la papila dental $(10,12,13,16,20)$.

Aunque el talón cuspídeo puede presentarse de forma aislada, también puede encontrarse asociado con sindromes como Morh, Sturge-Weber, Rubinstein-Taybi, hipomelanosis de Ito, Ellis-van Crevald y labio y paladar hendido; y con otras variaciones en la corona anatómica como incisivos laterales conoides, mesiodens, dens evaginatus en dientes posteriores, incisivos con forma de pala, dens invaginatus o tubérculo de Caravelli de crecimiento excesivo; aunquehayan sido reportadas anomalias como estas con talón cuspídeo, los casos de talón cuspídeo asociados con geminación son muy raros $(4,5,7,8,10,19,20)$.

El talón cuspídeo fue clasificado por Hattab et al en tres grados de acuerdo a su tamaño: a) tipo I o talón verdadero -estructura bien delimitada que se proyecta en la superficie palatina o lingual y se extiende por lo menos hasta la mitad de la distancia entre la unión esmalte-cemento y el borde incisal, b) tipo II o semitalón -reducción de tamaño del tipo anterior, que se extiende a menos de la mitad de la distancia entre la unión esmalte-cemento y el borde incisal, y c) tipo III o trazos de talón -forma de cíngulo pronunciado que no llega hasta el tercio medio de la superficie palatina o lingual, el cual puede ser redondeado, cónico o multilobulado (16, 20, 21, 22). Esta clasificación usa el mismo criterio que la definición dada para llamar a una estructura "talón cuspídeo", mientras que formaciones con forma de cúspide de menor tamaño en el área del cíngulo de incisivos son probablemente referidos de una mejor manera como "cíngulos agrandados” o “cíngulos prominentes”. A menudo, los talones cuspídeos presentan surcos de desarrollo prominentes o fisuras en las superficies laterales, donde se unen a la superficie lingual del diente (19).Los incisivos laterales superiores son los dientes que muestran más variaciones en cuanto a morfología y tamaño (20).

\section{Tratamiento de diente geminado con talón cuspídeo}

La geminación causa problemas estéticos, mal posicionamiento e impactación de los dientes adyacentes debido al mayor volumen de la corona del diente geminado $(7,8,9,11)$. Además, la presencia de un surco de desarrollo en algunos casos, hace a estos dientes susceptibles a lesión de caries y enfermedad periodontal $(2,11,16)$. Existen diversos enfoques de tratamiento, siendo la estética el factor determinante respecto a la decisión de conservar o extraer este diente. Una opción es el desgaste selectivo con el objetivo de reducir el ancho coronal, otra posible opción de tratamiento implica la extracción con posterior aproximación del diente adyacente y la modificación coronal con resina compuesta, es decir, reanatomización.Y, finalmente, una tercera opción es extraer este diente y sustituirlo con una prótesis fija dentosoportada o un implante (6).

Por otro lado, la presencia de talón cuspídeo no es una indicación de tratamiento dental a menos que cause problemas clínicos o estéticos (4).Dado que el principal problema clínico originado es irritación lingual, el manejo se encuentra dirigido a la remoción de la causa de esta (10). Un talón pequeño es usualmente asintomático y no necesita tratamiento, mientras que los talones cuspídeos grandes causan problemas clínicos incluyendo compromiso estético, interferencias 
oclusales, desplazamiento del diente afectado, irritación de la lengua durante el habla y la masticación, lesión de caries en los surcos de desarrollo que delinean las cúspides y atrición del diente antagonista. Además puede ocurrir necrosis pulpar, patología periapical, problemas periodontales asociados a excesivas fuerzas oclusales y dolor temporomandibular $(10,12,13,19)$. En cambio, las fisuras en las superficies laterales del talón deben ser restauradas en caso de lesión de caries, dado que tienen alta susceptibilidad a esta (19) .En caso de que se requiera, las alternativas de tratamiento incluyen un procedimiento de desgaste periódico aplicando un agente desensibilizante o una reducción de la cúspide con o sin tratamiento endodóntico(4,22).Gunduz y Acikgoz refieren que el talón cuspídeo debe ser reducido gradualmente en citas consecutivas con pausas de 6 a 8 semanas dando tiempo al depósito de dentina reparativa (5).

Dado que diversos informes relacionados con el examen radiográfico han indicado que los talones cuspídeos contienen cuernos pulpares en variadas extensiones, el tratamiento de los talones cuspídeos implica un juicio cuidadoso asociado ala presencia, dimensiones o ausencia de cuerno pulpar en las cúspides $(8,10)$. La vista radiográfica es inherentemente dificil debido a que la cúspide se superpone sobre la corona del diente afectado (10).

Actualmente han sido desarrolladas diversas resinas compuestas para procedimientos de alta estética, cuando son usadas apropiadamente, pueden realizarse restauraciones indistinguibles de la dentición natural. Los clínicos pueden reproducir la estética de un diente natural sin la necesidad de una preparación como la requerida para restauraciones indirectas de cerámica (23).

En la literatura no han sido hallados pasos específicos para realizar el desgaste en la reanatomización de un incisivo lateral geminado; sin embargo, existen pasos para la reanatomización de dientes caninos en incisivos laterales. El primer paso es desgastar el lóbulo vestibular, el segundo es desgastar en la zona incisal teniendo en cuenta la proporción prevista en el encerado de diagnóstico. Y finalmente, el tercer paso consiste en el desgaste lateralmente hacia cervical en el perfil de emergencia, abarcando la zona gingival tanto en la superficie mesial como distal (24).

\section{Reporte de Caso}

Paciente de sexo femenino de 21 años 4 meses de edad, refiere no padecer de enfermedad sistémica, acude al servicio de Odontología Restauradora y Estética de la Clínica de Posgrado de la Universidad Peruana Cayetano Heredia, con motivo de consulta: "Deseo verme mejor porque tengo un diente con forma extraña”. Asimismo, refiere no presentar historia de trauma orofacial ni antecedentes familiares de anomalías dentales.Sus dientes primarios erupcionaron a edad normal y no fueron notadas anormalidades en forma y tamaño en dientes deciduos.Al examen clínico extraoral paciente mesocéfalo, mesofacial, perfil convexo, normodivergente, con simetría facial conservada.

$\mathrm{Al}$ examen clínico intraoral ambos maxilares de forma ovoide (Figura 1) apiñados con relación molar clase III y relación canina clase I bilateral (Figuras 2 y 3), entrecruzamiento vertical de $20 \%$ y horizontal de $2 \mathrm{~mm}$. La corona del incisivo lateral izquierdo era más ancha en sentido mesio-distal que el incisivo contralateral (9,5 vs $7 \mathrm{~mm}$ ), presentaba un surco en el esmalte que corría en sentido vestíbulo-palatino cruzando el borde incisal produciendo una corona bífida (Figura 4); y además, presentaba una pronunciada y bien demarcada cúspide accesoria de forma piramidal en la superficie palatina, casi en fusión con la mitad distal de la corona bífida quedaba una apariencia de T observada desde una vista incisal. La cúspide anómala se extendía desde la unión cemento-adamantina pasando la mitad de la altura de la corona clínica hasta $1 \mathrm{~mm}$ antes del borde incisal. Un surco vertical en el borde distal del talón cuspídeo se extendía desde la base de la cúspide hasta unirse con el surco vestíbulo-palatino que divide la corona en dos mitades. La cúspide medía $6 \mathrm{~mm}$ en sentido mesio-distal y 5 en sentido incisivo-cervical, el ancho vestíbulo-palatino no podía ser medido debido a su unión con la mitad distal de la corona. El talón cuspídeo no interfiería con la oclusión ni causaba irritación lingual. A nivel del incisivo lateral contralateral, se observaba un cíngulo prominente de $2 \mathrm{~mm}$ de ancho en sentido vestíbulo-palatino y $1 \mathrm{~mm}$ en sentido incisivo-cervical que no alcanzaba la altura de la mitad de la corona, no interferia con la oclusión y no causaba irritación lingual. Ambas incisivos laterales medían $7 \mathrm{~mm}$ vestíbulo-lingualmente. 


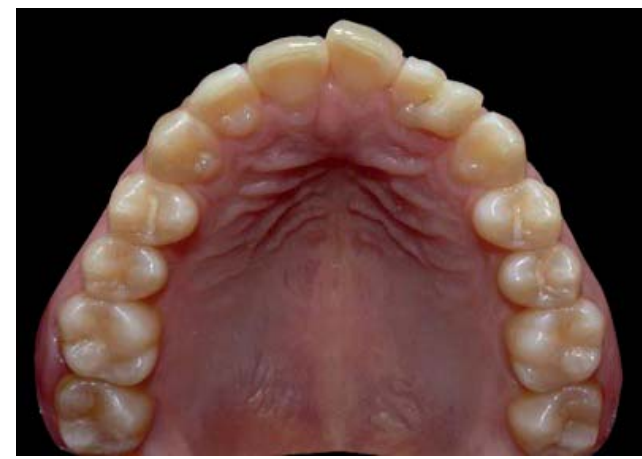

Figura 1. Vista oclusal antes del tratamiento.

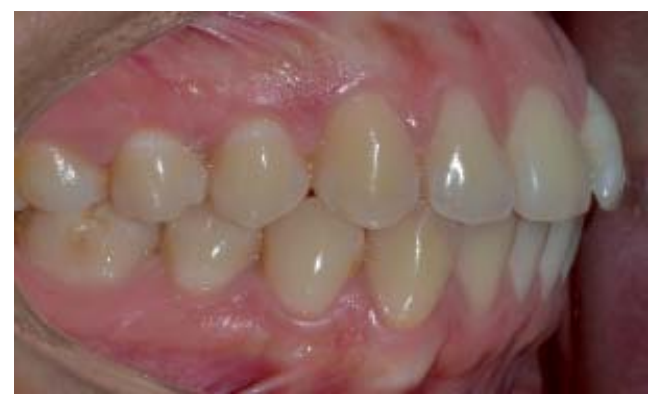

Figura 3. Vista lateral derecha antes del tratamiento.

En el análisis de modelos se observó que el borse inciso-distal del incisivo lateral izquierdo interfiere con la protrusión al contactar con la pieza 33 y con la guía canina del lado izquierdo al contactar con la pieza 32. En la radiografía periapical de la pieza 22 se observa alteración morfológica representada por una muesca coronal que da la apariencia de una corona bífida; asimismo, se observa la presencia de un solo conducto radicular y cámara pulpar. La unión de ambas coronas deja una zona radiolucida, probablemente una hendidura o fosa.

Se establece como diagnóstico definitivo: Paciente de sexo femenino de 21 años 4 meses; con perfil convexo, normodivergente, en dentición permanente, maloclusión clase III dentaria. Incisivo lateral superior derecho con talón lingual tipo III e incisivo lateral superior izquierdo geminado con talón lingual tipo I según la clasificación de Hattab y col., (16,20,21,22). A través de un análisis de modelos y encerado de diagnóstico se planificó hacer lo siguiente: Dado que la pieza 12 no interfiere con la guía anterior ni causa problema estético, se planificó hacer únicamente el sellado preventivo en la superficie palatina. Con la pieza 22, el objetivo es conseguir que la apariencia

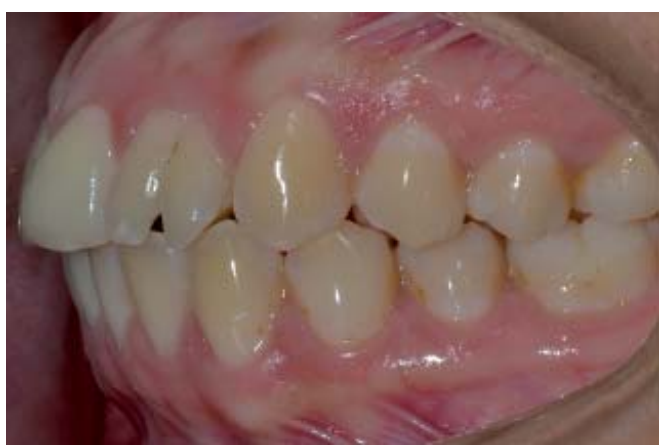

Figura 2. Vista lateral izquierda antes del tratamiento.

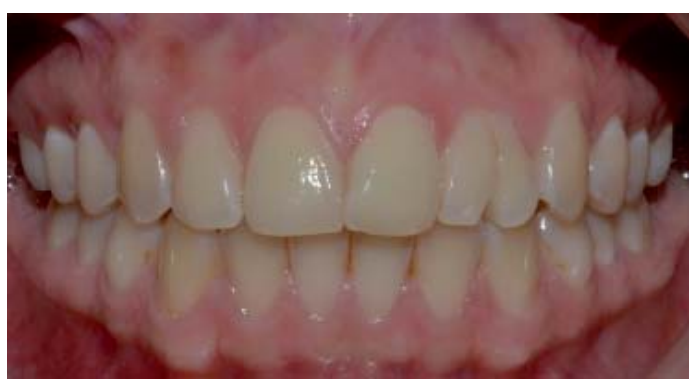

Figura 4. Vista frontal antes del tratamiento.

sea como la del incisivo contralateral, corregir la interferencia en protrusiva y lateralidad y mantener su posición en el arco con reanatomización coronal con resina compuesta.

Para conseguir una proporción dental adecuada, los desgastes realizados fueron similares a los que se usan para la reanatomización de dientes caninos en incisivos laterales según Hirata dado que el diente geminado presentaba 2 lóbulos, 2 cúspides incisales y un incremento de diámetro mesiodistal; sin embargo, se varió el orden de los pasos. Se inició con el desgaste lateralmente hacia cervical en el perfil de emergencia, abarcando la zona gingival tanto en la superficie mesial como distal. Utilizando puntas diamantadas de tipo fisura con punta redondeada en una pieza de mano de alta velocidad con abundante irrigación, se desgastó 1 y 1,5 mm en las superficies mesial y distal respectivamente, alcanzándose el mismo ancho mesiodistal de la pieza $12(7 \mathrm{~mm})$.

El desgaste proximal se realizó en dos citas periódicas a intervalo de 1 mes y con aplicación de barniz fluorado (Duraphat). En la primera cita se desgastó 0,5 mm en la superficie distal (Figura 5) y se colocó 
un espaciador interproximal en la superficie mesial de la pieza para facilitar el desgaste mesial programado para la siguiente cita (Figura 6); realizado esto, la paciente refirió no presentar sensibilidad. En la segunda cita se desgastó $1 \mathrm{~mm}$ más en la superficie distal y $1 \mathrm{~mm}$ en la superficie mesial, alcanzando el mismo ancho del incisivo contralateral (Figura 7).Completado este paso, se procedió con el desgaste de los dos

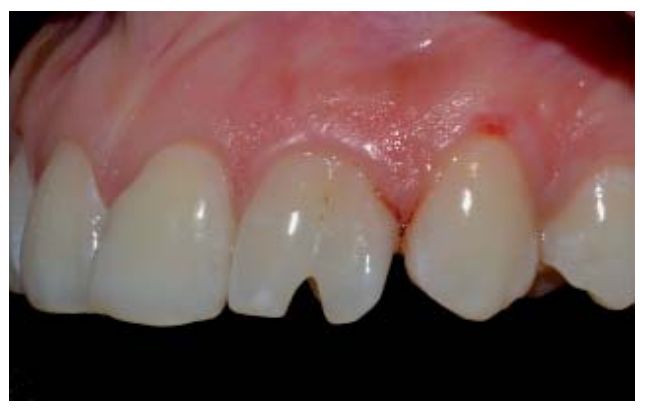

Figura 5. Desgaste de 0,5 mm en la superficie distal. lóbulos vestibulares separados por el surco vestibular. Y finalmente, se desgastó en la zona incisal teniendo en cuenta la proporción prevista en el encerado de diagnóstico.

Utilizando el encerado de diagnóstico se realizó una impresión con silicona de condensación a nivel de la pieza 22 para fabricar una guía de silicona.

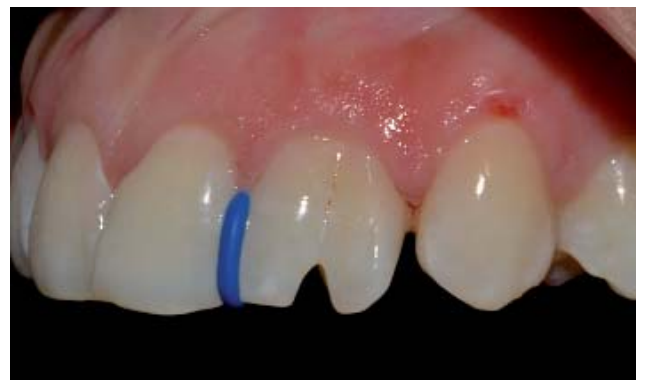

Figura 6. Colocación de liga interproximal.

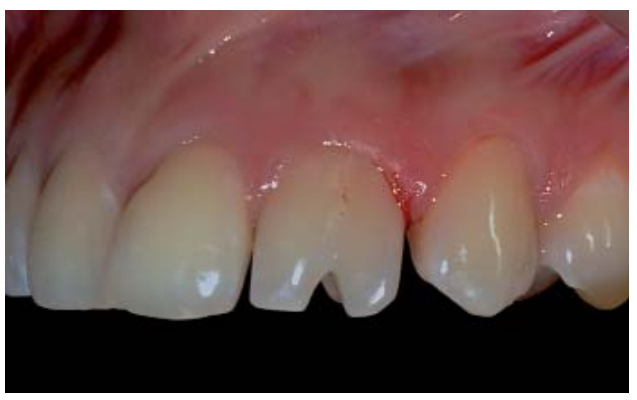

Figura 7. Desgaste de 0,5 mm en las superficies mesial y distal

Para darle a la pieza la anatomía de incisivo lateral, se inició con la inserción de resina compuesta (Filtek Z350 XT, 3M ESPE). Para dar el soporte paratino en la zona de la muesca central se aplicó resina Enamel A1 usando la guía de silicona, para la zona de la dentina se aplicó resina Body A2, para la capa del esmalte cromático fue usada resina Enamel A1, y la última capa, de esmalte acromático, se aplicó resina acromática Amelogen PlusEN (Ultradent)(Figuras 8 y 9). Posteriormente, se colocó un botón de resina compuesta polimerizada entre las piezas 21 y 22 para evitar la inclinación dental. Una semana después la paciente fue citada para realizar un control, en el cual se tomaron las últimas fotografías (Figuras 10, 11 y
12). Concluido el tratamiento planificado la paciente fue transferida al Servicio de Ortodoncia para una correcta distribución de espacios.

Los reportes que describen tratamiento para geminación son escasos e inconclusos $(11,12)$. En este caso, el objetivo fue reducir la estructura dental medio-distalmente y colocar una restauración con resina compuesta para mejorar la apariencia del paciente, mejorar la función y prevenir la acumulación de placa en la muesca de la corona (12). 


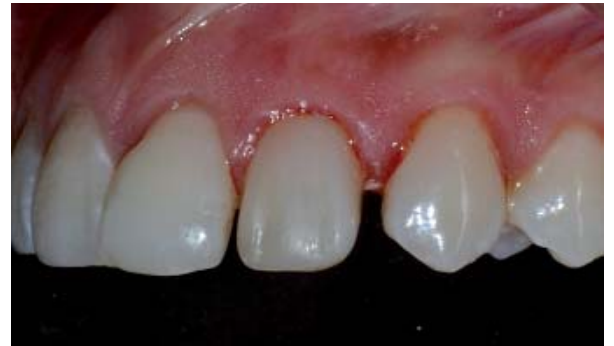

Figura 8. Carrilla directa realizada en la pieza 22

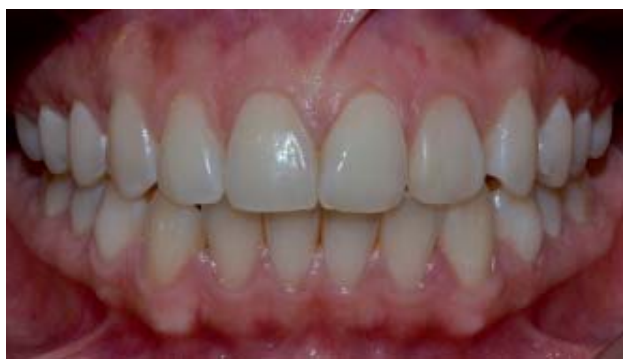

Figura 10. Vista frontal una semana después de rsealizar la carilla.

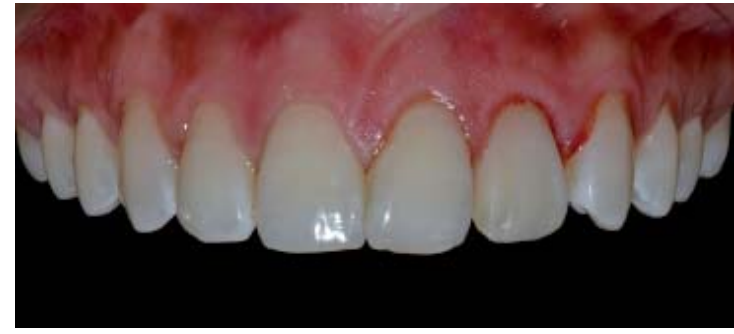

Figura 9. Vista frontal con contraste de la carrilla directa realizada

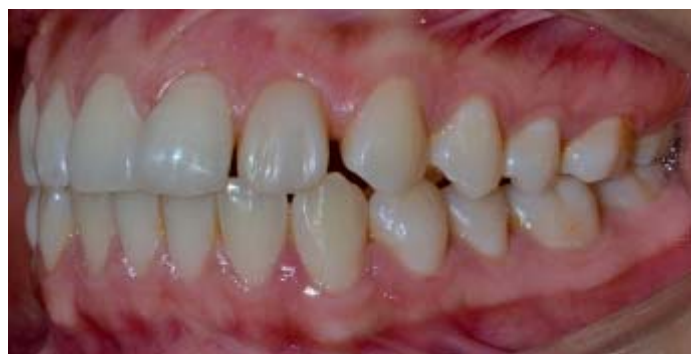

Figura 11. Vista lateral una semana después de realizar la carilla

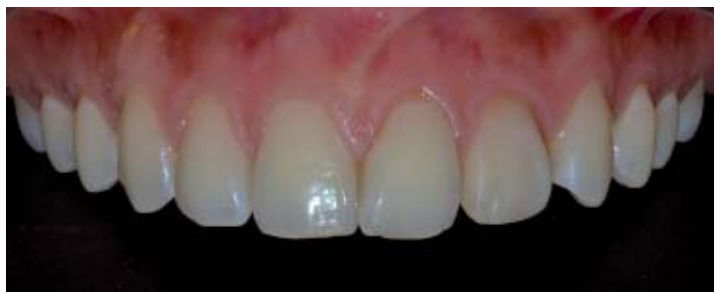

Figura 12. Vista frontal con contraste 1 semana después de realizar la carilla

\section{DISCUSIÓN}

En el presente caso, se considera como diagnóstico para la pieza 12 geminacióndebido ala presencia de un surco vestibular dando a la corona una apariencia bífida, la presenciade una raíz con un solo conducto y un conteo total correcto de dientes. Estos criterios fueron tomados en cuenta tambiénpor autores como Agnihotri y Rao $(2,6)$.

Por otro lado, el diagnóstico de talon cuspídeo está apoyado en los criterios de Hattab y col., y Mader, dado que la excresencia palatina se extiendea más de la mitad de la distancia entre la unión cemento-adamantina y el borde incisal( 5,19,21,22). Aunque Davis reporta que cuando un tubérculo se desvía, no siempre es necesario que alcance la distancia mencionada debido a que el tubérculo hace una inclinación hacia la superficie mesial $(5,25)$. Esto, a pesar de que autores como Gunduz K y Acikgoz A refieren que no existen criterios específicos para describir el talón cuspídeo y prefieren llamarlo“cíngulo hipertrófico" simplemente(5).

En relación a los criterios mencionados, el diagnósti- 
co de la pieza 12 fue: geminación con talón cuspídeo tipo I $(2,6,10,19)$. La presencia simultánea de geminación y talón cuspídeo en un mismo diente es un hallazgo muy raro $(8,4,6,13,16)$.

Los reportes de caso publicados de dientes permanentes geminados con talón cuspídeo hasta el momento son aquellos publicados por: Cullen (1985)(3), Al-Omari (1999)(10), Hattab (2001)(12), Cubukcu (2006)(4), Gunduz (2006)(5), Agnihotri (2007)(6), Tomazinho (2009)(7), Sener (2012)(8); y finalmente, Tewari (2013)(13). Entre estos, el único reporte en el que se ve afectado un incisivo lateral superior es en el reporte de Agnihotri (2007)(6), mientras que en los demás reportes los dientes afectados son incisivos centrales superiores.

Este es el primer caso reportado de incisivos laterales con talón cuspídeo bilaterales, uno de ellos geminado.

El manejo de dientes geminados con talón cuspídeo es variado. Autores como Hattab y Hazza'a (2001) (12) y Sener , Unlu, Basciftci y Bozdag (2012)(8) han decidido tomar medidas similares a la nuestra; es decir, reanatomizaciones con resina compuesta. Mientras que autores como Cullen y Pangrazio-Kulbersh (1985)(3), Cubukcu, Sonmez y Vedat (2006)(4), Gunduz y Acikgoz (2006) (5)y Agnihotri, Marwah y Goel (2007)(6) han optado por una medida más drástica, la exodoncia. Al-Omari et al., (1999) (10) se limitaron a la remoción del talón que provocaba la irritación lingual y Tomazinho, Baratto-Filho, Leonardi, Haragushiku y de Campos (2009)(7)únicamente han retirado lesión de caries con el consecuente tratamiento endodóncico.

La geminación y el talón lingual pueden generar problemas tanto clínicos como estéticos, por este motivo han recibido distintos tratamientos poco conservadores como exodoncia de la pieza dental para el caso de geminación o el desgaste y posterior endodoncia para el caso del talón lingual.

En el presente caso se optó por el tratamiento de reanatomización con resina compuesta por ser el más conservador y conllevar a una gran mejora de la apariencia del paciente sin sacrificar la pieza dental y tejido sano de piezas adyacentes. Este tratamiento debe ser controlado anualmente para el pulido de la resina compuesta.
El tratamiento por el que se optó tiene como ventajas que es de aplicación fácil, puede ser reparado, tiene bajo costo y al conservar la mayor cantidad de estructura dental sana, prolonga la vida media del diente.

\section{Correspondencia}

Louise Webb Linares

Correo Electrónico: louise.webb@upch.pe

\section{REFERENCIAS BIBLIOGRÁFICAS}

1. Alves KS, Cabral C, Almeida F, Coelho RM, Alves R. Anatomical Aspects of Permanent Geminate Superior Central Incisives. Int J Morphol. 2009; 27(2):515-17.

2. Rao PK, Veena KM, Chatra L, Shenai P. Twin tooth on either side: A case report of bilateral gemination. Ann Med Health Sci Res. 2013; 3(2):271-73.

3. Cullen CL, Pangrazio-Kulbersh V. Bilateral germination with talon's cusp: Report of case. J Am DentAssoc. 1985;111(1):58-9.

4. Cubukcu CE, Sonmez A, Vedat G. Labial and palatal talon cusps on geminated tooth associated with dental root shape abnormality: a case report. J Clin Pediatr Dent. 2006; 31(1):21-4.

5. Gunduz K, Acikgoz A. An unusual case of talon cusp on a geminated tooth. Braz Dent J. 2006; 17(4):3436.

6. Agnihotri A, Marwah N, Goel M. Geminated maxillary lateral incisor with talon's cusp: A rare case report. J Oral Health Comm Dent. 2007; 1(2):40-2.

7. Tomazinho FS, Baratto-Filho F, Leonardi DP, Haragushiku GA, de Campos EA. Occurrence of talon cusp on a geminated maxillary central incisor: a case report. J Oral Sci. 2009; 51(2): 297-300.

8. Sener S, Unlu N, Basciftci FA, Bozdag G. Bilateral geminated teeth with talon cusps: A case report. Eur J Dent. 2012; 6(4):440-4.

9. Kumar V, T. Ragavendra R, Deshmukh J, Vanka A, Kumar M, Kumar A. Endodontic treatment and esthetic management of a primary double tooth with direct composite using silicone buildup guide. Contemp Clin Dent. 2012; 3(S1):S92-S95.

10. Al-Omari MAO, Hattab FN, Darwazeh AMG, Dummer PMH. Clinical problems associated with unusual cases of talon cusp. Int Endod J. 1999;32(3):183-90.

11. Grover PS, Lorton L. Gemination and twinning in the permanent dentition. Oral Surg Oral Med Oral Pathol. 1985; 59(3): 313-8.

12. Hattab FN, Hazza'a AM. An unusual case of talon cusp on geminated tooth. J Can Dent Assoc. 2001; 67(5):263-6. 
13. Tewari T, Ather A, Ather H. Endodontic involvement of a geminated tooth with talon cusp: a rare ocurrence. J Mass Dent Soc. 2013; 62(2):46-7.

14. Neville BW, Damm DD, Allen CM, Bouquot JE. Patologia oral e maxilofacial. 2da edición. Rio de Janeiro: Editora Guanabara Koogan; 2004. p.789.

15. Brook AH, Winter GBW. Double teeth: A retrospective study of "geminated" and "fused" teeth in children. Br Dent J. 1970;129(3):123-30.

16. Nirmala S, Velpula L, Nuvvula S, Mallineni SK. An unusual occurrence of geminated primary tooth with talon's cusp. J Dr NTR Univ Health Sci. 2012;1(3):187-91.

17. Mitchell WH. Letter to the editor. Dental Cosmos. 1892; 34:1036.

18. Mellor J, Ripa L. Talon cusp a clinically significant anomaly. Oral Surg Oral Med Oral Pathol. 1970; 29(2):225-8.

19. Mader CL. Talon cusp. J Am Dent Assoc. 1981; 103(2):244-6.

20. Hernández J, Villavicencio J, Arce E, Moreno F. Talon cusp: a report of five cases. Rev FacOdontol Univ Antioq. 2010; 21(2):208-17.

21. Hattab FN, Yassin OM, al-Nimri KS. Talon cusp in permanent dentition associated with other dental anomalies: review of literature and report of seven cases. ASDC J Dent Child. 1996; 63(5):368-376.

22. Ozcelik B, Atila B. Bilateral palatal talon cusps on permanent maxillary lateral incisors: A case report. Eur J Dent. 2011;5(1):113-6.

23. Reis A, Higashi C, Loguercio AD. Re-anatomization of anterior eroded teeth by stratification with direct composite resin. J Esthet Restor Dent, 2009; 21(5):304-17.

24. Hirata R. TIPS. Claves en Odontología Estética. 1ra edición. Argentina: Editor Médica Panamericana; 2012.p. 325-35.

25. Davis PJ, Brook AH. The presentation of talon cusp: diagnosis, clinical features, associations and possible etiology. Br Dent J. 1986;160(3):84-8.

Recibido : 26/05/2015

Aceptado: 24/07/2015 